\title{
AN EXCESS OF MID-INFRARED EMISSION FROM THE TYPE Iax SN 2014dt
}

\author{
Ori D. Fox ${ }^{1}$, Joel Johansson ${ }^{2}$, Mansi Kasliwal ${ }^{3}$, Jennifer Andrews ${ }^{4}$, John Bally ${ }^{5}$, Howard E. Bond ${ }^{1,6}$, \\ Martha L. Boyer $^{7,8}$, R. D. Gehrz ${ }^{9}$, George Helou ${ }^{10}$, E. Y. Hsiao ${ }^{11}$, Frank J. Masci ${ }^{10}$, M. Parthasarathy ${ }^{12}$,

\begin{abstract}
Supernovae Type Iax (SNe Iax) are less energetic and less luminous than typical thermonuclear explosions. A suggested explanation for the observed characteristics of this subclass is a binary progenitor system consisting of a $\mathrm{CO}$ white dwarf primary accreting from a helium star companion. A single-degenerate explosion channel might be expected to result in a dense circumstellar medium (CSM), although no evidence for such a CSM has yet been observed for this subclass. Here we present recent Spitzer observations of the SN Iax 2014dt obtained by the SPIRITS program nearly one year post-explosion that reveal a strong mid-IR excess over the expected fluxes of more normal SNe Ia. This excess is consistent with $10^{-5} M_{\odot}$ of newly formed dust, which would be the first time that newly formed dust has been observed to form in a Type Ia. The excess, however, is also consistent with a dusty CSM that was likely formed in pre-explosion mass-loss, thereby suggesting a single degenerate progenitor system. Compared to other SNe Ia that show significant shock interaction (SNe Ia-CSM) and interacting corecollapse events (SNe IIn), this dust shell in SN 2014dt is less massive. We consider the implications that such a pre-existing dust shell has for the progenitor system, including a binary system with a mass donor that is a red giant, a red supergiant, or an asymptotic giant branch star.
\end{abstract}

Key words: circumstellar matter - dust, extinction - infrared: stars - supernovae: general - supernovae: individual (SN 2014dt)

\section{INTRODUCTION}

Type Ia supernovae ( $\mathrm{SNe}$ Ia) serve as precise cosmological distance indicators due to the ability to standardize their light curves (e.g., Phillips 1993). While the light curves can be standardized, questions remain regarding the homogeneity of the underlying progenitor systems. While the exploding primary star is generally accepted to be a $\mathrm{CO}$ white dwarf (WD) that experienced a thermonuclear explosion as it approached the Chandrasekhar limit, the nature of the companion donor star is ambiguous. Evidence now suggests both single-degenerate and double-degenerate channels (e.g., Maoz et al. 2014, and references within).

To further complicate the questions at hand, recent studies reveal a subsample of $\mathrm{SNe}$ Ia that are less energetic and less luminous than typical thermonuclear events (e.g., Foley et al. 2013, 2015, and references within). Designated the Type Iax subclass, these events exhibit ejecta with slower velocities than those of typical SNe Ia near maximum light. In contrast to normal $\mathrm{SNe}$ Ia, which likely undergo a deflagration that transitions into a detonation (Khokhlov 1991), the above characteristics of SNe Iax may suggest a full deflagration of a WD (Branch et al. 2004; Phillips et al. 2007), although some SNe Iax are interpreted as Chandrasekhar-mass explosions experiencing pulsationally delayed detonations (Stritzinger et al. 2015).
There is growing evidence to suggest that slow Type Ia supernovae may arise from single-degenerate systems. A UV pulse due to companion interaction was seen in iPTF14atg, a SN2002es-like supernova (Cao et al. 2015). Pre-explosion images of SN Iax 2012Z reveal a luminous blue source at the position of the SN, suggesting a non-degenerate He companion, although the data may also be interpreted as a massive primary star or an accretion disk around the exploding primary WD (McCully et al. 2014). Images of the SN Iax 2008ha four years post-explosion reveal a red source at the position of the $\mathrm{SN}$ that is consistent with either a thermally pulsing asymptotic giant branch (AGB) companion star or the bound remnant of the primary WD (Foley et al. 2014). These results are used to argue that SNe Iax must have a diverse set of progenitors (e.g., Foley et al. 2015; White et al. 2015).

The SN Iax 2014dt was discovered on 2014 October 29.838 in M61 (Nakano et al. 2014; Ochner et al. 2014). Pre-explosion imaging places upper-limits on the progenitor that are consistent with a CO WD primary and a non-degenerate $\mathrm{He}$ companion progenitor system, as was suggested for SN 2012Z, although perhaps with a slightly smaller and/or hotter donor (Foley et al. 2015). The pre-explosion data, however, are also consistent with a low-mass red giant (RG) or main-sequence star companion. If detected, a circumstellar medium (CSM) may be able to offer clues about the SN 2014dt progenitor 

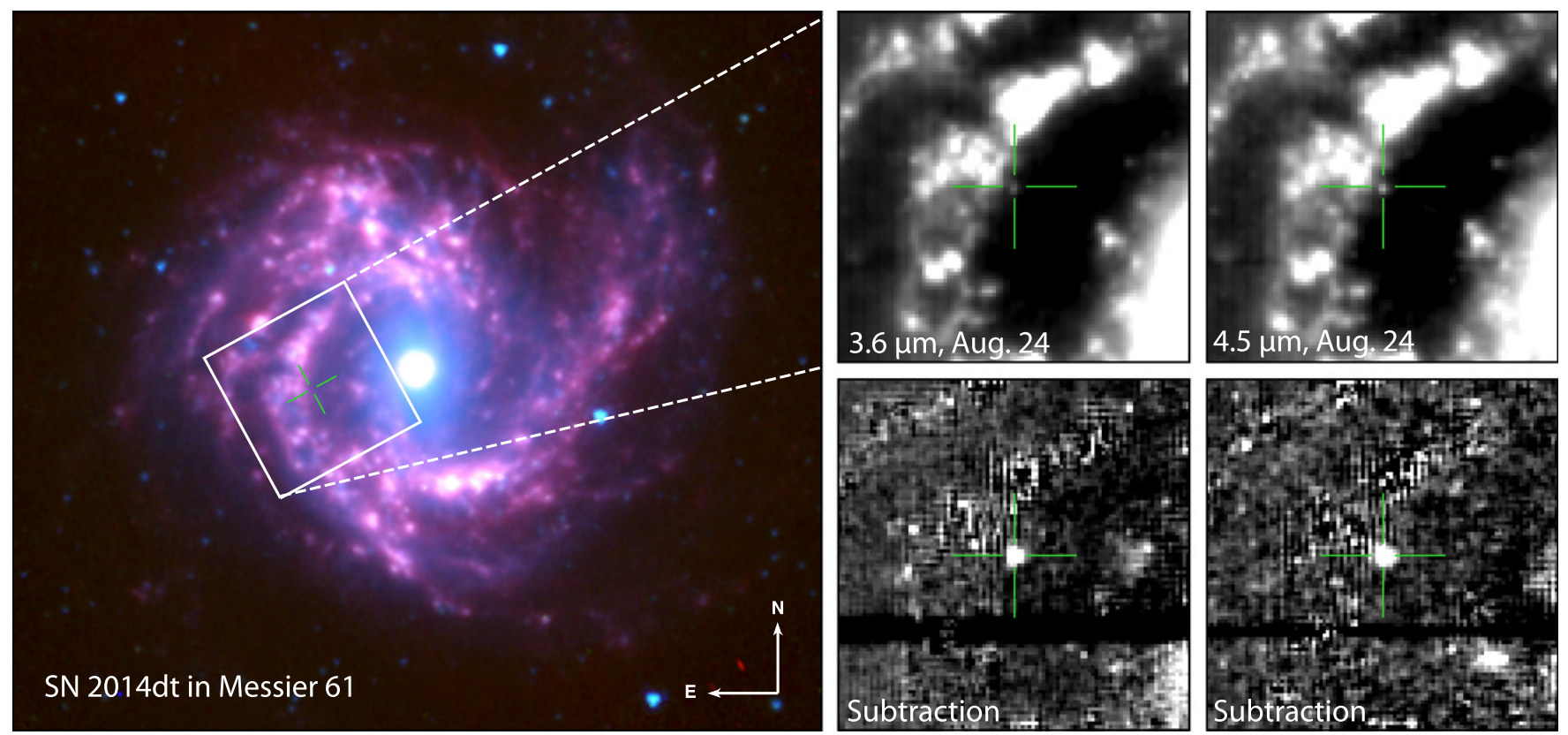

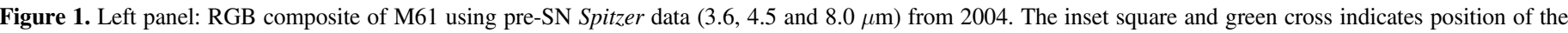

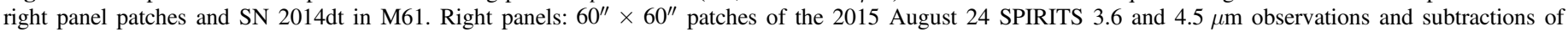
SN 2014dt.

system and pre-explosion mass-loss. For example, the presence of H-rich and dense CSM seen in the Type Ia-CSM subclass suggests a non-degenerate companion star in the progenitor system (e.g., Silverman et al. 2013; Fox et al. 2015). Narrow lines, often taken to be a key signature of a dense CSM, have not been previously observed in SNe Iax, but this result may be due to a lower density CSM or slower shock velocity. Like SNe Ia-CSM and their core-collapse counterparts (SNe IIn), another approach to detecting the presence of a CSM is through the presence of a mid-infrared (mid-IR) excess of thermal emission resulting from warm dust, in some cases years post-explosion (e.g., Fox et al. 2011; Fox \& Filippenko 2013).

In this Letter we present Spitzer Space Telescope (Werner et al. 2004; Gehrz et al. 2007) data of SN 2014dt obtained more than 300 days post-maximum by the SPitzer InfraRed Intensive Transients Survey (SPIRITS; Kasliwal et al. 2014). Section 2 lists the observational details. We use Spitzer photometry to constrain the dust mass, temperature, and IR luminosity. Section 3 considers the possible origin and heating mechanism of the dust. Section 4 summarizes the results and our conclusions.

\section{OBSERVATIONS}

\subsection{Warm Spitzer/IRAC Photometry}

Table 1 summarizes observations of SN 2014dt made by SPIRITS. This survey provides a systematic transient search of 194 nearby galaxies within $20 \mathrm{Mpc}$, on timescales ranging between a day to a year, to a depth of $20 \mathrm{mag}$ in the two Spitzer/IRAC (Fazio et al. 2004) channels at 3.6 and $4.5 \mu \mathrm{m}$. SPIRITS is an exploration science program that has been awarded $1130 \mathrm{hr}$ of Spitzer time over three years (2014-2016; PID \#11063, PI: Kasliwal). Concomitantly, the SPIRITS team undertakes extensive ground-based monitoring of these galaxies in the optical and near-infrared.
We obtained fully coadded Post Basic Calibrated Data (pbcd) from the Spitzer Heritage Archive (SHA). ${ }^{13}$ Figure 1 shows a combined 3.6, 4.5 and $8.0 \mu \mathrm{m}$ (RGB) image at a single epoch prior to the SN explosion. SPIRITS implements template subtraction to reduce photometric confusion from the underlying galaxy. ${ }^{14}$ Forced aperture photometry is then performed on the stack of IRAC co-add images for each channel. An aperture of fixed radius 4 pixels is centered on the position of SN2014dt. An aperture correction of $1.21 \times$ is applied to the total flux in the aperture for both channels. The background is computed using a median in an annulus with inner/outer radii of $8^{\prime \prime} / 15^{\prime \prime}$. Flux errors account for Poisson noise from the source and uncertainties in the local background estimate. Table 1 lists and Figure 2 plots the resulting photometry. Throughout the paper we use the zero magnitude fluxes for the IRAC Channels 1 and $2(\mathrm{CH} 1$ and $\mathrm{CH} 2$, with central wavelengths of 3.6 and $4.5 \mu \mathrm{m}$, respectively) of $F_{\nu, 0}^{\mathrm{CH} 1}=280.9 \mathrm{Jy}$ and $F_{\nu, 0}^{\mathrm{CH} 2}=179.7 \mathrm{Jy}$

\subsection{Optical and Near-IR Photometry}

Optical photometry were obtained with the Las Cumbres Observatory Global Telescope (LCOGT) Network (PIs: J. Bally, E. Gomez, and K. Finkelstein) in BVR and ri filters. The data were reduced and analyzed with standard $\operatorname{IRAF}^{15}$ routines, using the QUBA pipeline (see Valenti et al. 2011 for details). Template galaxy subtraction is not performed. The SN magnitudes are measured with a point-spread function (PSF) fitting technique (using daophot) and calibrated using SDSS

\footnotetext{
${ }^{13}$ SHA can be accessed from http://sha.ipac.caltech.edu/applications / Spitzer/SHA/.

${ }^{14}$ http://web.ipac.caltech.edu/staff/fmasci/home/mystats / ApPhotUncert.pdf

${ }^{15}$ IRAF: The Image Reduction and Analysis Facility is distributed by the National Optical Astronomy Observatory, which is operated by the Association of Universities for Research in Astronomy (AURA) under cooperative agreement with the National Science Foundation (NSF).
} 
Table 1

Spitzer Observations and IR Fitting Parameters ( $a=0.1 \mu \mathrm{m}$ Amorphous Carbon)

\begin{tabular}{|c|c|c|c|c|c|c|}
\hline $\begin{array}{l}\text { JD } \\
-2,450,000\end{array}$ & $\begin{array}{l}\text { Epoch } \\
\text { (days) }\end{array}$ & $3.6 \mu \mathrm{m}^{\mathrm{a}}$ & $4.5 \mu \mathrm{m}^{\mathrm{a}}$ & $M_{\mathrm{d}}\left(M_{\odot}\right)$ & $T_{\mathrm{d}}(\mathrm{K})$ & $L_{\mathrm{d}}\left(L_{\odot}\right)$ \\
\hline 7259 & 309 & $49(16)$ & $52(12)$ & $1.35 \times 10^{-5}$ & 711 & $4.75 \times 10^{5}$ \\
\hline 7267 & 317 & $52(18)$ & $58(13)$ & $1.83 \times 10^{-5}$ & 679 & $4.98 \times 10^{5}$ \\
\hline 7286 & 336 & 75 (18) & 73 (14) & $1.33 \times 10^{-5}$ & 770 & $7.48 \times 10^{5}$ \\
\hline
\end{tabular}

Note.

${ }^{\mathrm{a}} 1 \sigma$ uncertainties are given in parentheses.

photometry of stars in the field. For the light curve analysis we also include gri and $R$-band photometry from the Palomar 48 and 60-inch telescopes, $U B V$ photometry ${ }^{16}$ from the UltraViolet/Optical Telescope (UVOT, Roming et al. 2005) on the Swift spacecraft (Gehrels et al. 2004) and measurements by amateurs. ${ }^{17}$ In order to match the SN 2014dt photometry, the RI data were converted to $r i$ magnitudes using the conversions in Jordi et al. (2006).

Near-IR (NIR) observations in the Mauna Kea Observatory $J H K_{s}$ filters were carried out with the United Kingdom Infrared Telescope (UKIRT). Template galaxy subtraction is not performed. PSF fitting photometry was performed on the skysubtracted frames, calibrated using 2MASS stars in the field. We also include the early NIR photometry from Joshi et al. (2014).

\subsection{Distance to M61}

Figure 2 plots the multi-wavelength light curve of SN 2014dt compared to other SNe Iax 2005k and 2012Z. We note that SN 2014dt was discovered after peak brightness, but the light curves indicate that it peaked around October 20 $(\mathrm{MJD}=56,950)$. This explosion date is comparable to the time of maximum deduced from spectral cross correlations (Ochner et al. 2014; Foley et al. 2015). Two different methods based on the Type II SN 2008in (Roy et al. 2011) yield distances to M61 ranging between a distance modulus $\mu=30.45 \mathrm{mag}$ (12.3 Mpc, EPM method, Bose \& Kumar 2014) and $\mu=31.43 \mathrm{mag}$ (19.3 Mpc, Photospheric magnitude method, Rodríguez et al. 2014). While the former is consistent with the Tully-Fisher estimate (Schoeniger \& Sofue 1997) and used to put limits on the progenitor system in Foley et al. (2015), the larger distance makes SN 2014dt appear to have similar absolute magnitudes as SNe 2005hk and 2012Z, peaking at $M_{V} \sim-18 \mathrm{mag}$. We assume a distance of 19.3 Mpc for the analysis in this paper.

\subsection{A Mid-IR Excess}

Both SN 2012Z (Stritzinger et al. 2015; Yamanaka et al. 2015) and SN 2005hk (Phillips et al. 2007; Sahu et al. 2008) had low host-galaxy extinction $E(B-V) \leqslant 0.10$, and SN 2014dt seems to follow the early color evolution of both these SNe. However, at around 100 days post peak SN 2014dt starts to exhibit redder colors than SNe 2005hk and 2012Z. As we explain below, the redder colors likely stem from additional flux at longer wavelengths, rather than dust attenuation of bluer light.

\footnotetext{
${ }^{16}$ Made public by Peter Brown on the Swift Supernovae page (http://people. physics.tamu.edu/pbrown/SwiftSN/swift_sn.html).

17 Made public on the AAVSO (https://www.aavso.org/apps/webobs/ results $/$ ?star $=\mathrm{SN}+2014 \mathrm{DT}$ ) and the Bright Supernovae (http://www. rochesterastronomy.org/sn2014/sn2014dt.html) pages.
}

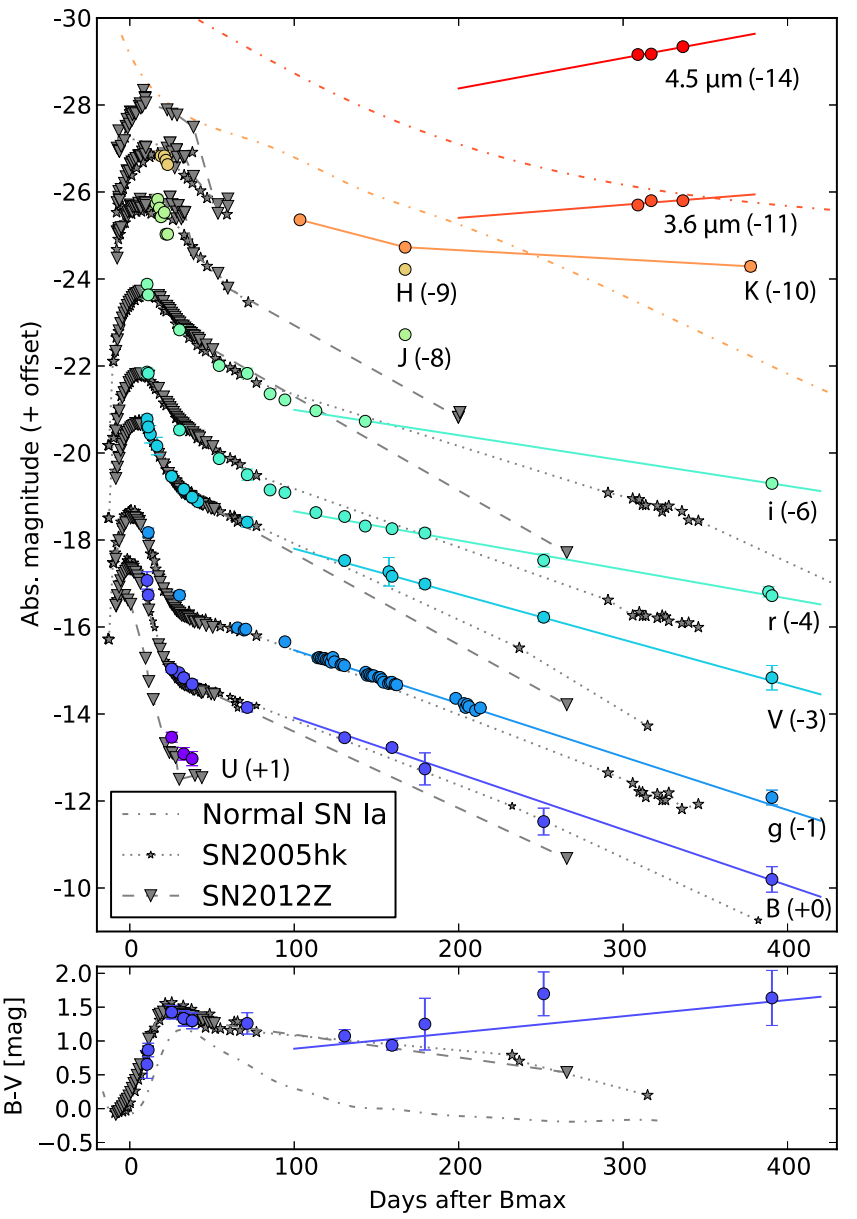

Figure 2. Upper panel: optical, near- and mid-IR light curves of SN 2014dt (colored circles and solid lines) assuming a distance modulus $\mu=31.43 \mathrm{mag}$ and $\mathrm{MJD}_{B, \max }=56,950$. The light curves of SNe 2005hk (gray stars and dotted lines; Phillips et al. 2007; Sahu et al. 2008) and 2012Z (gray triangles and dashed lines; Stritzinger et al. 2015; Yamanaka et al. 2015) are shown for comparison as well as the 3.6 and $4.5 \mu \mathrm{m}$ templates for normal SNe Ia (dashed-dotted lines) from Johansson et al. (2014). Bottom panel: $B-V$ color evolution of SN 2014dt compared to a normal SN Ia and SNe Iax 2005hk and $2012 Z$.

Figure 2 shows that SN 2014dt has plateaued between days 298 and 326. Such behavior is not typical of thermonuclear SNe Ia, which follow radioactive decay rates at these epochs, even in the mid-IR (Johansson et al. 2014). For a comparison, Figure 3 plots the SN 2014dt Spitzer Channel 2 photometry over a mid-IR template compiled for both normal $\mathrm{SNe}$ Ia (Johansson et al. 2014) and a sample of SNe with observed dust present. SN 2014dt shows a clear mid-IR excess in both Spitzer bands. 


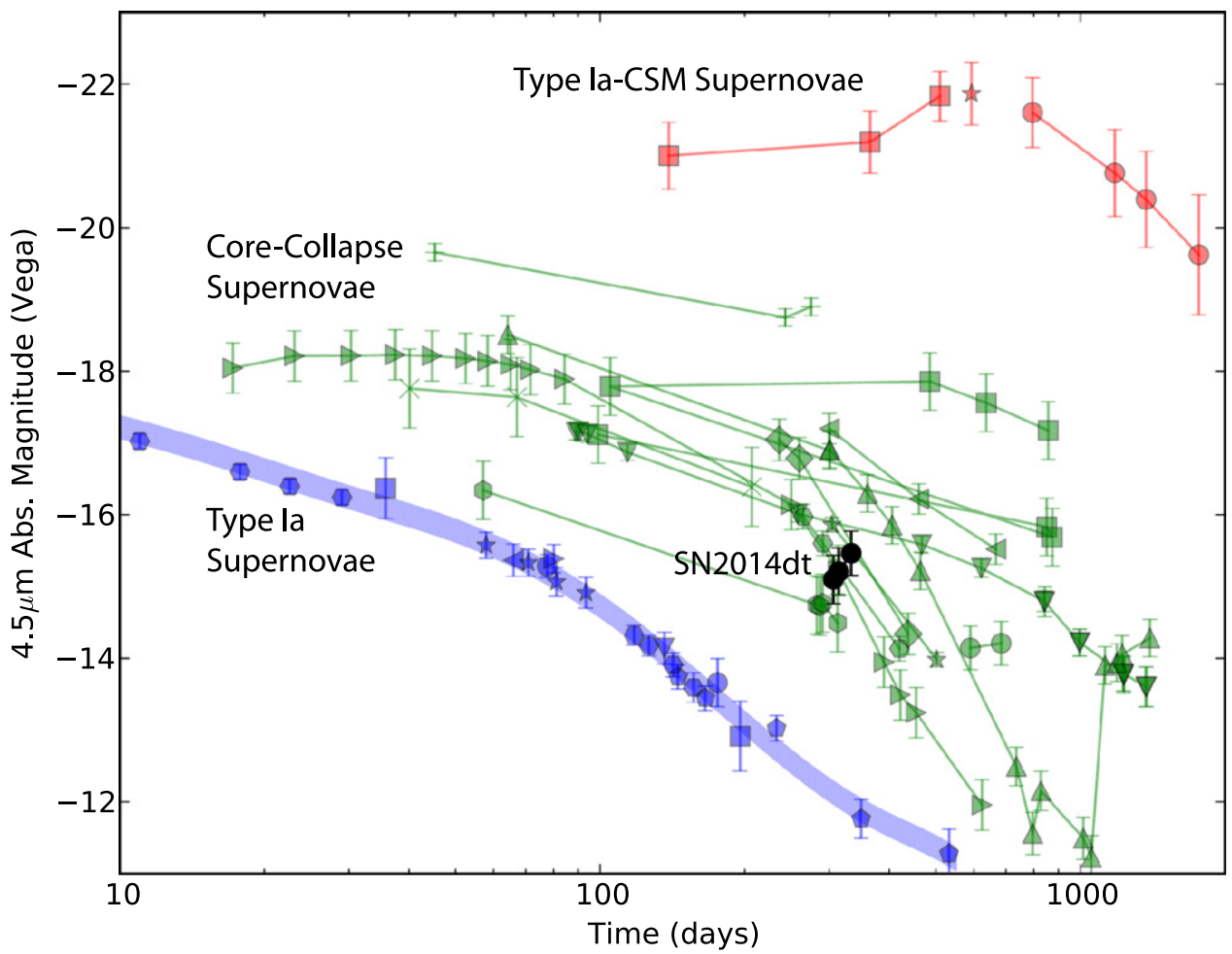

Figure 3. Collage of $4.5 \mu \mathrm{m}$ absolute magnitudes of normal SNe Ia (blue symbols, from Johansson et al. 2014), core-collapse SNe (green symbols, from Szalai \& Vinkó, 2013; S. Tinyanont et al. 2015, in preparation, and references therein) and strongly interacting SNe Ia-CSM (red symbols, from Fox et al. 2011 and Fox \& Filippenko 2013).

At late times, SN Ia light curves transition from a regime dominated by gamma-rays to one dominated by positrons. In the case of energy deposition by positrons, the IR light curve slope can change due to various physical effects that govern the positron escape probability (e.g., magnetic fields; Penney \& Hoeflich 2014). For a normal SN Ia at 300 days, this effect is only on the order of a tenth of a magnitude. We note that even if the positrons are fully trapped, this effect cannot explain the observed IR plateau.

The source of the mid-IR excess is therefore likely to be warm dust. Spitzer photometry offers the advantage of spanning the peak of the warm dust grain blackbody emission. The SED can be fit as a function of the dust mass, $M_{\mathrm{d}}$, and temperature, $T_{\mathrm{d}}$,

$$
F_{\nu}=\frac{M_{\mathrm{d}} B_{\nu}\left(T_{\mathrm{d}}\right) \kappa_{\nu}(a)}{d^{2}}
$$

Where $a$ is the dust grain radius and $d$ is the distance of the dust from the observer. Here we assume optically thin dust emitting at a single equilibrium temperature (e.g., Hildebrand 1983), where $\kappa_{\nu}(a)$ is the dust absorption coefficient. An optically thick case is beyond the scope of this paper.

The dust composition and temperature distribution is unknown. Given only 2 photometry points, however, we assume a simple dust population of a single size and temperature composed entirely of amorphous carbon (AC; Williams \& Fox 2015). These assumptions are consistent with previous analysis of warm Spitzer data (e.g., Fox et al. 2011; Fox \& Filippenko 2013), which allows for meaningful comparisons. Figure 4 and Table 1 present the best fitting (i.e., minimized value of $\chi^{2}$ ) dust mass and temperature obtained for SN 2014dt at each epoch. Given only two photometry points at each epoch, we do not consider the presence of more than one dust component. The dust masses presented here are likely lower limits since a bulk of the dust likely resides at cooler temperatures not probed by Spitzer. For a distance to M61 of only 12 Mpc, however, both the dust mass and luminosity presented in Table 1 would decrease by a factor of $\sim 2.5 \times$, but the mid-IR excess relative to the normal SN Ia sample would still be present.

Compared to the mid-IR excess observed in the SNe Ia-CSM 2002ic and 2005gj (e.g., Fox \& Filippenko 2013), the dust luminosity (and mass) of SN 2014dt is approximately two orders of magnitude smaller than the SNe Ia-CSM, while the temperatures are about the same (but note the fit is made with only two photometry points). We explore the implications of these similarities and differences below.

\section{ANALYSIS AND DISCUSSION}

\subsection{The Source of the Mid-IR Emission}

Given that the mid-IR fluxes in Figures 2 and 3 fall significantly above the expected radioactive decay light curves of normal SNe Ia, we attribute a majority of the mid-IR flux to warm dust. The dust, however, may be either newly formed or pre-existing, and heating may be collisional or radiative (see Fox et al. 2010 for a full discussion). To constrain the origin and heating mechanism, we first calculate the size of the dust shell. The minimum radius is given by the blackbody radius, $r_{\mathrm{bb}}=\left[L_{d} /\left(4 \pi \sigma T_{d}^{4}\right)\right]^{1 / 2}$, where we assume a spherically symmetric distribution and an optically thick shell. The integrated IR luminosity $L_{d} \approx 5 \times 10^{5} L_{\odot}$ and dust temperature $T_{\mathrm{d}} \approx 700$ $\mathrm{K}$ yield a $r_{\mathrm{bb}} \approx 3.5 \times 10^{15} \mathrm{~cm}$. A caveat that should be considered is that the dust is likely optically thin since SN 


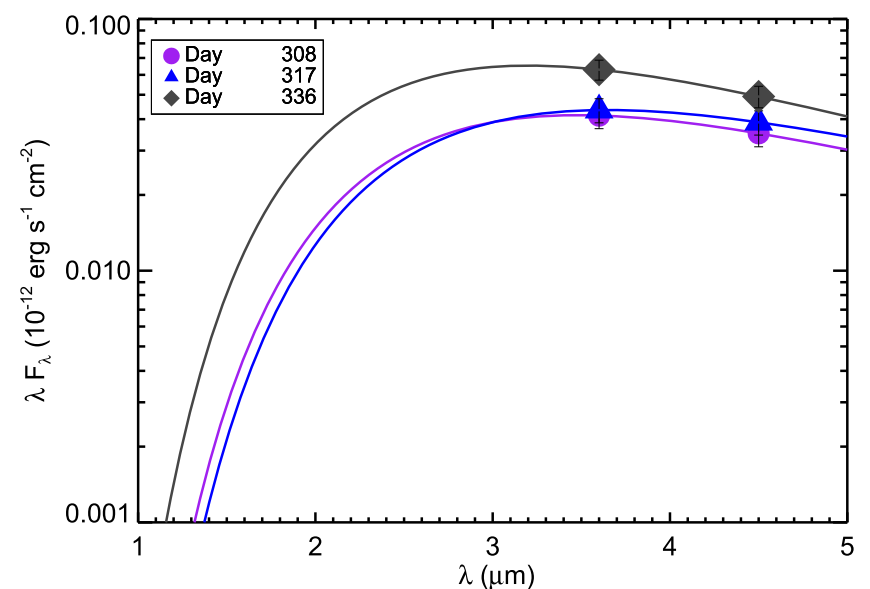

Figure 4. Photometry of SNe 2014dt in Spitzer/IRAC Channels $1(3.6 \mu \mathrm{m})$ and $2(4.5 \mu \mathrm{m})$. Overplotted are the resulting best fits of Equation (1).

2014t does not exhibit much extinction, so the dust shell radius is likely larger than the calculated blackbody radius or the dust is asymmetrically distributed.

In any case, the blackbody radius is comparable to the distance traveled by material with a time averaged velocity of $\sim 1300 \mathrm{~km} \mathrm{~s}^{-1}$ over $\sim 300$ days. Indeed, models suggest dust can form in $\mathrm{SNe}$ Ia at these velocities, timescales, and total mass (Nozawa et al. 2011). Furthermore, SNe Iax are defined by their low velocities (e.g., Foley et al. 2013, and references within). It is interesting to note that no other SN Ia has ever been observed to form new dust (and this paper presents the first late-time mid-IR observations of any SN Iax). Simultaneous optical and IR data would be beneficial since newly formed dust is often associated with not only a rise in the IR luminosity, but also a simultaneous drop in the visual luminosity due to absorption. Unfortunately, our optical photometry do not extend to the epochs of the Spitzer observations.

Another possibility that is consistent with the data is preexisting dust in the CSM. Figure 8 in Fox et al. (2010) shows that the minimum radius derived above is approximately consistent with the distance to which such dust would have been vaporized (assuming a vaporization temperature of 2000 $\mathrm{K}$ ) by the peak luminosity of SN $2014 \mathrm{dt}(M \approx-18 \mathrm{mag}$; Foley et al. 2014). A dust shell lying at the vaporization radius is usually a signature of continuous mass loss from the progenitor primary (or companion) since a temporary mass-loss scenario (i.e., eruption) would have to include a contrived timeline. For the case of pre-existing dust, the heating mechanism could be an IR echo, shocks, or radiation from the X-ray/UV/optical emission produced by shock interaction at an inner radius. An IR echo at this radius would last only a few days $\left(t \approx \frac{2 r}{c}\right)$, so the duration of the mid-IR light curve rules out that scenario. Shock heating of the dust, however, is possible given that the $\sim 1300 \mathrm{~km} \mathrm{~s}^{-1}$ shock radius corresponds to both the derived blackbody and vaporization radii.

Alternatively, radiative heating of a pre-existing dust shell by $\mathrm{X}$-ray/UV/optical emission generated by the shock interaction has been proposed to explain the late-time IR emission observed in SNe IIn and Ia-CSM (e.g., Fox et al. 2011, 2013; Fox \& Filippenko 2013). Assuming an optically thin dust shell, the observed dust temperature $\left(T_{\mathrm{d}}\right)$ and shell radius $\left(r_{\mathrm{d}}\right)$ require a combined optical, ultraviolet, and/or X-ray flux given by

$$
L_{\mathrm{opt} / \mathrm{UV} / \mathrm{X}}=\frac{64}{3} \rho a r_{\mathrm{d}}^{2} \sigma T_{\mathrm{SN}}^{4} \frac{\int B_{\nu}\left(T_{\mathrm{d}}\right) \kappa(\nu) d \nu}{\int B_{\nu}\left(T_{\mathrm{SN}}\right) Q_{\mathrm{abs}}(\nu) d \nu}
$$

where $T_{\mathrm{SN}}$ is the effective SN blackbody temperature, $\rho$ is the dust bulk (volume) density, $\kappa(\nu)$ is the dust absorption coefficient, and $Q_{\mathrm{abs}}$ is the dust absorption efficiency. For SN $2014 \mathrm{dt}$, the $T_{\mathrm{d}} \approx 700 \mathrm{~K}$ dust at $r_{\mathrm{bb}} \approx 3 \times 10^{15} \mathrm{~cm}$ requires a radiative heating source in the range $L_{\mathrm{opt} / \mathrm{UV} / \mathrm{X}} \approx 10^{7} L_{\odot}$ (see Figure 4 of Fox et al. 2013). Optical/UV/and X-ray observations could confirm the predicted luminosities. Furthermore, narrow lines in the optical spectra would offer clear evidence of CSM interaction. As already noted, our optical photometry do not extend to the epochs of the Spitzer observations, and we have no optical spectroscopy at these later epochs to search for narrow lines (the original classification spectrum does not show evidence of narrow lines at early times; Foley et al. 2015).

\subsection{Progenitor Mass-loss Rate}

Mid-IR wavelengths probe the characteristics of the CSM at the dust-shell radius. Assuming a dust-to-gas mass ratio expected in the H-rich envelope of a massive star, $Z_{\mathrm{d}}=M_{\mathrm{d}} / M_{\mathrm{g}} \approx 0.01$, the dust-shell mass can be tied to the progenitor's total mass-loss rate,

$$
\begin{aligned}
\dot{M}= & \frac{M_{\mathrm{d}}}{Z_{\mathrm{d}} \Delta r} v_{\mathrm{w}} \\
= & \frac{3}{4}\left(\frac{M_{\mathrm{d}}}{M_{\odot}}\right)\left(\frac{v_{\mathrm{w}}}{120 \mathrm{~km} \mathrm{~s}^{-1}}\right) \\
& \times\left(\frac{5 \times 10^{16} \mathrm{~cm}}{r}\right)\left(\frac{r}{\Delta r}\right)\left[M_{\odot} \mathrm{yr}^{-1}\right],
\end{aligned}
$$

for a progenitor wind speed $v_{\mathrm{w}}$. Since we do not know the pre$\mathrm{SN}$ wind speed, we assume a speed of $\sim 10 \mathrm{~km} \mathrm{~s}^{-1}$. If we also assume a thin shell, $\Delta r / r=1 / 10$, Equation (3) yields a massloss rate of $<10^{-6}\left(v_{\mathrm{w}} / 10 \mathrm{~km} \mathrm{~s}^{-1}\right) M_{\odot} \mathrm{yr}^{-1}$, which is only an upper limit since the blackbody radius is only a lower limit. This mass-loss rate is consistent with either a RG or red supergiant (RSG; Gehrz \& Woolf 1971; Drout et al. 2015) or an AGB star (Marshall et al. 2004).

\section{SUMMARY}

This Letter presents new Spitzer data on the SN Iax 2014dt obtained nearly one year post-discovery. The mid-IR data show evidence of emission from warm dust. The warm-dust parameters are consistent with both newly formed dust and a pre-existing dust shell that is either heated by the forward shock or radiatively heated by optical, UV, and X-ray emission generated by the shock at interior radii. In the former case, this would be the first documented case of newly formed dust observed in a Type Ia (Nozawa et al. 2011 and references therein). Alternatively, newly formed dust may be suggestive of a core-collapse origin. Indeed, Valenti et al. (2009) argue that $\mathrm{SNe}$ Iax could be core-collapse events that are both lowenergy and hydrogen deficient due to a massive star collapsing into a black hole. In the latter case, the pre-existing CSM suggests a non-degenerate companion star. This CSM has a 
derived mass-loss rate of $<10^{-6} M_{\odot} \mathrm{yr}^{-1}$. Such a mass-loss rate is nearly a factor $10 \times$ smaller than the Type Ia-CSM PTF11kx (Dilday et al. 2012), which may explain both the lack of absorption and shock interaction observed in the earliest spectrum (Foley et al. 2015). Without knowledge of the pre-SN wind speed, these mass-loss rates have a number of degeneracies when it comes to the progenitor system, including a RG, RSG, or even a AGB star. Although not considered in the original paper (Foley et al. 2014), such a dusty CSM may also be the source of the red emission observed in the vicinity of SN 2008ha. While pre-SN mass loss often suggests a singledegenerate channel, a caveat should be noted that such massloss rates have also been derived for both the core-degenerate and double-degenerate models (e.g., Shen et al. 2013; Soker et al. 2013). Future multi-wavelength observations of SNe Iax, and SN 2014dt in particular, will be necessary to disentangle the various scenarios.

This work is based on data obtained via Program \#11063 with the Spitzer Space Telescope, which is operated by the Jet Propulsion Laboratory, California Institute of Technology, under a contract with NASA. Support for this work was provided by NASA through an award issued by JPL/Caltech. The authors thank Ryan Foley for useful discussions. We thank Peter Milne, Jacob Jencson, and Tom Prince for help with observations.

\section{REFERENCES}

Bose, S., \& Kumar, B. 2014, ApJ, 782, 98

Branch, D., Baron, E., Thomas, R. C., et al. 2004, PASP, 116, 903

Cao, Y., Kulkarni, S. R., Howell, D. A., et al. 2015, Natur, 521, 328

Dilday, B., Howell, D. A., Cenko, S. B., et al. 2012, Sci, 337, 942

Drout, M. R., Milisavljevic, D., Parrent, J., et al. 2015, ApJ, submitted (arXiv:1507.02694)

Fazio, G. G., Hora, J. L., Allen, L. E., et al. 2004, ApJS, 154, 10

Foley, R. J., Challis, P. J., Chornock, R., et al. 2013, ApJ, 767, 57

Foley, R. J., McCully, C., Jha, S. W., et al. 2014, ApJ, 792, 29
Foley, R. J., van Dyk, S. D., Jha, S. W., et al. 2015, ApJL, 798, L37 Fox, O. D., Chevalier, R. A., Dwek, E., et al. 2010, ApJ, 725, 1768 Fox, O. D., Chevalier, R. A., Skrutskie, M. F., et al. 2011, ApJ, 741, 7 Fox, O. D., \& Filippenko, A. V. 2013, ApJL, 772, L6

Fox, O. D., Filippenko, A. V., Skrutskie, M. F., et al. 2013, AJ, 146, 2 Fox, O. D., Silverman, J. M., Filippenko, A. V., et al. 2015, MNRAS, 447, 772 Gehrels, N., Chincarini, G., Giommi, P., et al. 2004, ApJ, 611, 1005 Gehrz, R. D., Roellig, T. L., Werner, M. W., et al. 2007, RScI, 78, 1302

Gehrz, R. D., \& Woolf, N. J. 1971, ApJ, 165, 285

Hildebrand, R. H. 1983, QJRAS, 24, 267

Johansson, J., Goobar, A., Kasliwal, M. M., et al. 2014, MNRAS, submitted (arXiv:1411.3332)

Jordi, K., Grebel, E. K., \& Ammon, K. 2006, A\&A, 460, 339

Joshi, V., Srivastava, M., Banerjee, D. P. K., Venkataraman, V., \& Ashok, N. M. 2014, ATel, 6772, 1

Kasliwal, M. M., Tinyanont, S., Jencson, J., et al. 2014, ATel, 6644, 1

Khokhlov, A. M. 1991, A\&A, 245, 114

Maoz, D., Mannucci, F., \& Nelemans, G. 2014, ARA\&A, 52, 107

Marshall, J. R., van Loon, J. T., Matsuura, M., et al. 2004, MNRAS, 355, 1348

McCully, C., Jha, S. W., Foley, R. J., et al. 2014, Natur, 512, 54

Nakano, S., Itagaki, K., Guido, E., et al. 2014, CBET, 4011, 1

Nozawa, T., Maeda, K., Kozasa, T., et al. 2011, ApJ, 736, 45

Ochner, P., Tomasella, L., Benetti, S., et al. 2014, CBET, 4011, 2

Penney, R., \& Hoeflich, P. 2014, ApJ, 795, 84

Phillips, M. M. 1993, ApJL, 413, L105

Phillips, M. M., Li, W., Frieman, J. A., et al. 2007, PASP, 119, 360

Rodríguez, Ó, Clocchiatti, A., \& Hamuy, M. 2014, AJ, 148, 107

Roming, P. W. A., Kennedy, T. E., Mason, K. O., et al. 2005, SSRv, 120,95

Roy, R., Kumar, B., Benetti, S., et al. 2011, ApJ, 736, 76

Sahu, D. K., Tanaka, M., Anupama, G. C., et al. 2008, ApJ, 680, 580

Schoeniger, F., \& Sofue, Y. 1997, A\&A, 323, 14

Shen, K. J., Guillochon, J., \& Foley, R. J. 2013, ApJL, 770, L35

Silverman, J. M., Nugent, P. E., Gal-Yam, A., et al. 2013, ApJS, 207, 3

Soker, N., Kashi, A., García-Berro, E., Torres, S., \& Camacho, J. 2013 MNRAS, 431, 1541

Stritzinger, M. D., Valenti, S., Hoeflich, P., et al. 2015, A\&A, 573, A2

Szalai, T., \& Vinkó, J. 2013, A\&A, 549, 79

Valenti, S., Fraser, M., Benetti, S., et al. 2011, MNRAS, 416, 3138

Valenti, S., Pastorello, A., Cappellaro, E., et al. 2009, Natur, 459, 674

Werner, M. W., Roellig, T. L., Low, F. J., et al. 2004, ApJS, 154, 1

White, C. J., Kasliwal, M. M., Nugent, P. E., et al. 2015, ApJ, 799, 52

Williams, B. J., \& Fox, O. D. 2015, ApJL, 808, L22

Yamanaka, M., Maeda, K., Kawabata, K. S., et al. 2015, ApJ, 806, 191 